\title{
WELL-POSEDNESS AND BLOW-UP FOR AN INHOMOGENEOUS SEMILINEAR PARABOLIC EQUATION
}

\author{
MOHAMED MAJdouB
}

Abstract. We consider the large-time behavior of sign-changing solutions of the inhomogeneous equation $u_{t}-\Delta u=|x|^{\alpha}|u|^{p}+\zeta(t) \mathbf{w}(x)$ in $(0, \infty) \times \mathbb{R}^{N}$, where $N \geqslant 3, p>1, \alpha>-2, \zeta$, w are continuous functions such that $\zeta(t)=t^{\sigma}$ or $\zeta(t) \sim t^{\sigma}$ as $t \rightarrow 0, \zeta(t) \sim t^{m}$ as $t \rightarrow \infty$. We obtain local existence for $\sigma>-1$. We also show the following:

- If $m \leqslant 0, p<\frac{N-2 m+\alpha}{N-2 m-2}$ and $\int_{\mathbb{R}^{N}} \mathbf{w}(x) d x>0$, then all solutions blow up in finite time;

- If $m>0, p>1$ and $\int_{\mathbb{R}^{N}} \mathbf{w}(x) d x>0$, then all solutions blow up in finite time;

- If $\zeta(t)=t^{\sigma}$ with $-1<\sigma<0$, then for $u_{0}:=u(t=0)$ and $\mathbf{w}$ sufficiently small the solution exists globally.

We also discuss lower dimensions. The main novelty in this paper is that blow up depends on the behavior of $\zeta$ at infinity.

Mathematics subject classification (2010): 35K05, 35A01, 35B44.

Keywords and phrases: Inhomogeneous parabolic equation, global existence, finite time blow-up, differential inequalities, forcing term depending of time and space, critical Fujita exponent.

\section{REFERENCES}

[1] J. Aguirre And J. Giacomoni, The shape of blow-up for a degenerate parabolic equation, Differ. Integral Equ., 14 (2001), 589-604.

[2] B. Ben Slimene, S. TAYAChi And F. B. Weissler, Well-posedness, global existence and large time behavior for Hardy-Hénon parabolic equations, Nonlinear Analysis, 152 (2017), 116-148.

[3] C. BAndle, H. A. Levine AND Qi S. ZHANG, Critical Exponents of Fujita Type for Inhomogeneous Parabolic Equations and Systems, Journ. of Math. Anal. and App., 251 (2000), 624-648.

[4] K. Deng AND H. A. Levine, The role of critical exponents in blowup theorems, the sequel, J. Math. Anal. Appl., 243 (2000), 85-126.

[5] J. DiXON AND S. MCKeF, Weakly singular discrete Gronwall inequalities, Z. angew. Math. Mech., 64 (1986), 535-544.

[6] M. J. ESTEBAN AND J. GiACOMONI, Existence of global branches of positive solutions for semilinear elliptic degenerate problems, J. Math. Pures Appl., 79 (2000), 715-740.

[7] H. FujitA, On the blowing up of solutions of the Cauchy problem for $u_{t}=\Delta u+u^{1+\alpha}$, J. Fac. Sci. Univ. Tokyo Sec. IA Math. 13 (1966), 109-124.

[8] V. A. Galaktionov and J. L. VÁzQUez, The problem of blow-up in nonlinear parabolic equations, Discrete Contin. Dyn. Syst., 8 (2002), 399-433.

[9] J. Giacomoni, Some results about blow-up and global existence to a semilinear degenerate heat equation, Rev. Mat. Complut., 11 (1998), 325-351.

[10] Y. HAN, Blow-up phenomena for a reaction diffusion equation with special diffusion process, Applicable Analysis (2020), doi:10.1080/00036811.2020.1792447.

[11] K. HAYAKAWA, On nonexistence of global solutions of some semilinear parabolic differential equations, Proc. Japan Acad. 49 (1973), 503-505.

[12] B. Hu, Blow Up Theories for Semilinear Parabolic Equations, Springer, Berlin (2011).

[13] M. JLeli, T. KAWAKAMI AND B. SAMET, Critical behavior for a semilinear parabolic equation with forcing term depending of time and space, J. Math. Anal. Appl. 486 (2020), 123931. 
[14] O. A. Ladyzenskaja, V. A. Solonnikov And N. N. Uralćeva, Linear and quasilinear equations of parabolic type, Amer. Math. Soc., Transl. Math. Monographs, Providence, R. I. (1968).

[15] H. A. LEVINE, The role of critical exponents in blowup theorems, SIAM Rev., 32 (1990), 269-288.

[16] H. A. LEVINE AND P. MEIER, The value of the critical exponent for reaction-diffusion equations in cones, Arch. Rational Mech. Anal., 109 (1989), 73-80.

[17] A. V. Martynenko AND A. F. Tedeev, Cauchy problem for a quasilinear parabolic equation with a source term and an inhomogeneous density, Comput. Math. Math. Phys., 47 (2007), 238-248.

[18] A. V. Martynenko AND A. F. Tedeev, On the behavior of solutions to the Cauchy problem for a degenerate parabolic equation with inhomogeneous density and a source, Comput. Math. Math. Phys., 48 (2008), 1145-1160.

[19] E. Mitidieri AND S. I. PohoZAev, A priori estimates and blow-up of solutions of nonlinear partial differential equations and inequalities, Proc. Steklov Inst. Math., 234 (2001), 3-383.

[20] C. A. STUART, A critically degenerate elliptic Dirichlet problem, spectral theory and bifurcation, Nonlinear Analysis, 190 (2020), Article ID 111620.

[21] Z. TAN, Reaction-diffusion equations with special diffusion processes, Chin. J. Contemp. Math., 22 (2001), 371-382; translation from Chin. Ann. Math., Ser. A, 22 (2001), 597-607.

[22] G. D. Yomgne And M. Majdoub, Blow up and global existence of solutions to a Hardy-Hénon equation with a spatial-temporal forcing term, submitted.

[23] YUAN-WEI QI, The critical exponents of parabolic equations and blow-up in $\mathbb{R}^{n}$, Proceedings of the Royal Society of Edinburgh, 128A (1998), 123-136.

[24] P. QuitTner And P. Souplet, Superlinear parabolic problems, Birkhäuser Verlag, Basel (2007), xii+584.

[25] S. TAYACHI, Uniqueness and non-uniqueness of solutions for critical Hardy-Hénon parabolic equations, J. Math. Anal. Appl., 488 (2020), 123976.

[26] Q. S. ZHANG, A new critical phenomenon for semilinear parabolic problem, J. Math. Anal. Appl., 219 (1998), 123-139.

[27] Q. S. ZHANG, Blow up and global existence of solutions to an inhomogeneous parabolic system, J. Differential Equations, 147 (1998), 155-183.

[28] X. WAng, On the Cauchy problem for reaction-diffusion equations, Transactions of the American Mathematical Society, 337 (1993), 549-590. 